\title{
Interior a priori estimates for the Monge-Ampère equation
}

\author{
Jiakun Liu and Xu-Jia Wang
}

\begin{abstract}
In this paper we prove the strict convexity, the interior $C^{1, \alpha}$, $C^{2, \alpha}$ and $W^{2, p}$ estimates for convex solutions to the Monge-Ampère type equation. For the strict convexity and $C^{1, \alpha}$ estimate, we assume that the inhomogeneous term $f$ satisfies a doubling condition. For the $C^{2, \alpha}$ and $W^{2, p}$ estimates, we assume that $f$ is Hölder continuous or continuous. These estimates are mainly due to Caffarelli. We also give a brief discussion on the regularity for more general Monge-Ampère type equations arising in optimal transportation.
\end{abstract}

\section{Introduction}

In this paper we consider the regularity of solutions to the MongeAmpère equation

$$
\operatorname{det} D^{2} u=f \quad \text { in } \Omega \text {, }
$$

where $\Omega$ is a domain in $\mathbb{R}^{n}$, det $D^{2} u$ denotes the determinant of the Hessian matrix $D^{2} u$, and $f$ is a given function. This is a fully nonlinear, second order partial differential equation. It is elliptic when the Hessian matrix $D^{2} u$ is positive definite, namely when $u$ is locally uniformly convex. In this paper we always assume that the solution is convex, and the inhomogeneous term $f$ is nonnegative.

When $f=K\left(1+|D u|^{2}\right)^{(n+2) / 2}$, equation (1.1) is the prescribed Gauss curvature equation, with the Gauss curvature $K$. The Monge-Ampère equation also arises in a variety of applications, such as in affine geometry, isometric embedding, optimal transportation, and reflector design. It has been extensively studied in the last century. Significant contributions were made by Aleksandrov, Calabi, Pogorelov, Heinz, Caffarelli-Nirenberg-Spruck, ChengYau, Krylov, and many others before 1990s. In particular Pogorelov proved

2010 Mathematics Subject Classification. 35B45, 35J96.

Key words and phrases. Monge-Ampère equations, $C^{2, \alpha}$ estimate, $W^{2, p}$ estimate. Both authors were supported by the Australian Research Council.

(C) 2015 International Press 
an interior second derivative estimate for strictly convex solutions and Calabi obtained an estimate for higher derivatives. Therefore a regularity theory in the case when $f$ is positive and smooth has been established. We refer the reader to $[\mathbf{7}, \mathbf{8}, \mathbf{9}, \mathbf{1 5}, \mathbf{1 6}, \mathbf{1 7}, \mathbf{2 9}, \mathbf{3 2}, \mathbf{3 3}]$ for more details.

In this paper we address the interior a priori estimates for the MongeAmpère equation, mainly obtained by Caffarelli in 1990s $[\mathbf{3}, \mathbf{4}, \mathbf{5}]$. But in dimension two, these estimates were obtained earlier $[\mathbf{1 7}, \mathbf{2 8}, \mathbf{3 0}]$. We will discuss the following estimates:

a) Strict convexity of solutions;

b) $C^{1, \alpha}$ regularity of solutions;

c) Continuity of the second derivatives;

d) Hölder continuity of the second derivatives;

e) $W^{2, p}$ estimate for large $p$;

f) $W^{2,1+\epsilon}$ estimate for small $\epsilon>0$.

For part a), we assume that the solution vanishes on the boundary and $f$ satisfies a doubling condition. The strict convexity of solutions is assumed in all the estimates b)-f). For estimate b), we assume that $f$ satisfies a doubling condition. For estimates c) and d), we assume that $f$ is positive and Dini or Hölder continuous. For estimate e), we assume that $f$ is positive and continuous. By an example in [35], the continuity is needed for the $W^{2, p}$ estimate for large $p$. But for small $p>1$, it suffices to assume that $c_{0}<f<c_{1}$ for some positive constants $c_{0}$ and $c_{1}$, and that is the estimate in $\mathrm{f})$.

Among the above estimates, the main ones are the $C^{2, \alpha}$ and $W^{2, p}$ estimate by Caffarelli [4]. However, the paper [4] is difficult to understand. For example no details for the $C^{2, \alpha}$ were given. Even for the uniformly elliptic equation, the proof of the $C^{2, \alpha}$ is quite complicated [2]. A simplified proof for the $C^{2, \alpha}$ estimate was later given in [19], using a perturbation argument introduced in [37]. In [37] a simple proof for the $C^{2, \alpha}$ estimate for elliptic and parabolic equations, both linear and nonlinear, was presented. For the $W^{2, p}$ estimate, the proof in this paper is also simpler than that in [4], but the basic idea is the same as that in [4]. The purpose of this article is to provide simplified proof for Caffarelli's work on the Monge-Ampère equation. The presentation of this paper is based on the lectures given by the second author at Tsinghua University in 2011. In this note we also provide a simple proof for the existence and uniqueness of minimum ellipsoids of convex bodies.

This paper is organized as follows. In Section 2, we discuss the strict convexity and $C^{1, \alpha}$ regularity of solutions. In Section 3 we outline the proof in [19] for a continuity estimate for the second derivatives of solutions. In Section 4, following the lines of [4] with some simplification in [38], we give a shorter proof of the $W^{2, p}$ estimate, assuming $f$ is continuous. When $f$ is not continuous but pinched by two positive constants, recently De PhilippisFigalli-Savin [12] and Schmidt [30] proved $u \in W_{\text {loc }}^{2,1+\varepsilon}$ for some small $\varepsilon>0$. We include the proof of the $W_{\mathrm{loc}}^{2,1+\varepsilon}$ estimate in Section 5. Finally in Section 
6 we briefly discuss the corresponding estimates for the more general MongeAmpère equation

$$
\operatorname{det}\left[D^{2} u-A(x, u, D u)\right]=f(x, u, D u) .
$$

This more general equation arises in reflector design and optimal transportation. The interior $C^{2, \alpha}$ and $W^{2, p}$ estimates for (1.2) have also been obtained recently in $[\mathbf{2 3}, \mathbf{2 4}]$, respectively, under some appropriate assumptions on the cost function.

\section{Strict convexity and $C^{1, \alpha}$ estimate}

\subsection{Minimum ellipsoid.}

First we introduce a lemma, which is due to F. John, and is often used in the study of convex bodies and the Monge-Ampère equation.

Lemma 2.1. Let $\Omega$ be a bounded, convex domain in $\mathbb{R}^{n}$. Then among all ellipsoids containing $\Omega$, there is a unique ellipsoid $E$ of smallest volume such that

$$
\frac{1}{n} E \subset \Omega \subset E .
$$

In this paper we denote by $\alpha \Omega$ the $\alpha$-dilation of $\Omega$ with respect to the center of its minimum ellipsoid. We call $E$ the minimum ellipsoid of $\Omega$. By a rotation of the coordinates, we may assume that $E$ is given by

$$
E=\left\{\sum_{i=1}^{n}\left(\frac{x_{i}-x_{0, i}}{r_{i}}\right)^{2}<1\right\}
$$

where $x_{0}=\left(x_{0,1}, \cdots, x_{0, n}\right)$. By the unimodular linear transform $T: x \rightarrow y$,

$$
y_{i}=\frac{r}{r_{i}}\left(x_{i}-x_{0, i}\right)+x_{0, i}, \quad i=1, \cdots, n,
$$

where $r=\left(r_{1} \cdots r_{n}\right)^{1 / n}, E$ becomes the ball $B_{r}\left(x_{0}\right)$ with

$$
B_{r / n}\left(x_{0}\right) \subset T(\Omega) \subset B_{r}\left(x_{0}\right) .
$$

We say $\Omega$ is normalized if its minimum ellipsoid is a ball (namely when $T$ is the identity mapping).

Proof of Lemma 2.1. Let $V_{0}=\inf \{|E|: E \in \Phi\}$, where $\Phi$ is the set of ellipsoids containing $\Omega$. Let $E_{k}$ be a sequence of ellipsoids in $\Phi$ with $\left|E_{k}\right| \rightarrow V_{0}$. Since $E_{k}$ contains $\Omega$, it must be uniformly bounded and converges in Hausdorff distance to an minimum ellipsoid $E$.

To show that $E$ satisfies (2.1), we assume by a linear transform that $E$ is the unit sphere with center at the origin. If (2.1) is not true, let $x_{0} \in \partial \Omega$ such that $\left|x_{0}\right|=\inf _{x \in \partial \Omega}|x|$. By a rotation of axes, we assume $x_{0}=(0, \cdots, 0,-t)$ with $t \leq \frac{1}{n}-\epsilon$ for some $\epsilon>0$, such that the plane $\left\{x_{n}=-t\right\}$ is a tangent plane of $\partial \Omega$ at $x_{0}$. Then we have $\Omega \subset G=: B_{1}(0) \cap\left\{x_{n}>-t\right\}$. It suffices to prove that the unit ball is not the minimum ellipsoid of $G$. 
The proof is very elementary. Let $y_{i}=x_{i} /(1+\delta)$ for $i=1, \cdots, n-1$, and $y_{n}=x_{n}(1+\delta)^{n-1}$, where $\delta=\epsilon^{2}$. In the new coordinates, $G$ is strictly contained in the unit sphere with center at $(0, \cdots, 0, h)$ provided $\epsilon$ is sufficiently small, where $h=(1+\delta)^{n-1}-1+\delta^{2}$. We reach a contradiction as $E$ is a minimum ellipsoid.

Next, we prove the uniqueness. Suppose there exist two minimum ellipsoids $E_{1}$ and $E_{2}$. By a rescaling we assume $E_{1}=\left\{\sum x_{i}^{2}<1\right\}$ and $E_{2}=\left\{\sum\left(x_{i}-a_{i}\right)^{2} / r_{i}^{2}<1\right\}$ with $\prod r_{i}=1$, where $\left(a_{1}, \cdots, a_{n}\right)$ is the centre of $E_{2}$. Then for any $x \in E_{1} \cap E_{2}$, we have

$$
\sum_{i}\left[x_{i}^{2}+\frac{\left(x_{1}-a_{i}\right)^{2}}{r_{i}^{2}}\right]<2 .
$$

That is

$$
\sum_{i} \frac{1+r_{i}^{2}}{2 r_{i}^{2}}\left[\left(x_{i}-\frac{a_{i}}{1+r_{i}^{2}}\right)+\frac{a_{i}^{2} r_{i}^{2}}{\left(1+r_{i}^{2}\right)^{2}}\right]<1
$$

namely,

$$
\sum_{i} \frac{1+r_{i}^{2}}{2 r_{i}^{2}}\left(x_{i}-\frac{a_{i}}{1+r_{i}^{2}}\right)^{2}<1-\sum_{i} \frac{a_{i}^{2}}{2\left(1+r_{i}^{2}\right)} \leq 1 .
$$

Hence the domain $\Omega \subset E_{1} \cap E_{2}$ is contained in the ellipsoid

$$
E=\left\{\sum_{i} \frac{1+r_{i}^{2}}{2 r_{i}^{2}}\left(x_{i}-\frac{a_{i}}{1+r_{i}^{2}}\right)^{2}<1-\sum_{i} \frac{a_{i}^{2}}{2\left(1+r_{i}^{2}\right)}\right\} .
$$

Note that $\prod r_{i}=1$. Hence

$$
\prod_{i=1}^{n} \frac{1+r_{i}^{2}}{2 r_{i}^{2}}=\prod_{i=1}^{n} \frac{1}{2}\left(1+r_{i}^{2}\right) \geq 1
$$

which implies that $|E| \leq\left|E_{1}\right|$ and the equality achieves if and only if $a_{i}=0$ and $r_{i}=1$ for all $i=1, \cdots, n$. Therefore, we obtain the uniqueness.

The above proof of the uniqueness seems new, and is much simpler than the known ones. The uniqueness of the minimum ellipsoid is not needed in our treatment below. We remark that there is also a unique ellipsoid contained in $\Omega$ with maximal volume among all ellipsoids contained in $\Omega$.

\subsection{Uniform and Hölder estimates}

The rest part of this section is devoted to the strict convexity and $C^{1, \alpha}$ estimate. The presentation is similar to that in [33]. Consider the MongeAmpère equation,

$$
\operatorname{det} D^{2} u=\nu \text { in } \Omega
$$

where $\Omega$ is a bounded, convex domain in $\mathbb{R}^{n}, \nu$ is a finite measure. 
THEOREM 2.1. (Uniform estimate) Let $u$ be a bounded, convex solution to (2.6) subject to the boundary condition $u=0$ on $\partial \Omega$. If

$$
\nu(\Omega) \leq b \nu\left(\frac{1}{2} \Omega\right)
$$

for some positive constant $b$, then

$$
C^{-1}\{|\Omega| \nu(\Omega)\}^{1 / n} \leq \sup |u| \leq C\{|\Omega| \nu(\Omega)\}^{1 / n},
$$

where $C$ is a constant depending only on $n$ and $b$.

In particular if $\nu=f d x$ and $c_{0} \leq f \leq c_{1}$ for positive constants $c_{0}, c_{1}$, then

$$
C^{-1}|\Omega|^{2 / n} \leq \sup |u| \leq C|\Omega|^{2 / n}
$$

where $C$ depends only on $n, c_{0}, c_{1}$.

Proof. Let $E=\left\{\sum\left(x_{i}-a_{i}\right)^{2} / r_{i}^{2}<1\right\}$ be the minimum ellipsoid of $\Omega$. The Monge-Ampère equation is affine invariant and homogeneous, by making the changes $x_{i} \mapsto \frac{x_{i}-a_{i}}{r_{i}}$ and $u \mapsto u / h$, where $h=\left[\left(r_{1} \cdots r_{n}\right) \nu(\Omega)\right]^{1 / n}$, we may assume that

$$
\begin{gathered}
B_{1 / n}(0) \subset \Omega \subset B_{1}(0), \\
\nu(\Omega)=1, \quad \nu\left(\frac{1}{2} \Omega\right) \geq b^{-1} .
\end{gathered}
$$

To prove (2.7), it suffices to prove that $C^{-1} \leq \sup |u| \leq C$.

To prove $\sup |u| \leq C$, we use the assumption $\nu(\Omega)=1$. Assume that $\sup |u|$ attains at $\hat{x} \in \Omega$. Let $w$ be a convex function vanishing on $\partial \Omega$ and its graph is a convex cone with vertex at $(\hat{x}, u(\hat{x}))$. By convexity, $N_{w}(\Omega) \subset N_{u}(\Omega)$ and $B_{r}(0) \subset N_{w}(\Omega)$ for $r=\frac{1}{2} \sup |u|$ as $\Omega \subset B_{1}(0)$, where $N_{u}$ is the normal mapping of $u[\mathbf{3 3}]$. Hence sup $|u|^{n} \leq C\left|N_{w}(\Omega)\right| \leq C$.

To prove $\sup |u| \geq C$, we use the assumption $\nu\left(\frac{1}{2} \Omega\right) \geq b^{-1}$. For any $x \in \frac{1}{2} \Omega$,

$$
|D u(x)| \leq \frac{-\inf u}{\operatorname{dist}(x, \partial \Omega)} \leq 2 n \sup |u|
$$

Hence $\nu\left(\frac{1}{2} \Omega\right)=\left|N_{u}\left(\frac{1}{2} \Omega\right)\right| \leq C \sup |u|^{n}$.

TheOREM 2.2. (Global Hölder estimate) Let u be a generalized solution to

$$
\begin{aligned}
\operatorname{det} D^{2} u & =\nu & & \text { in } \Omega, \\
u & =\varphi & & \text { on } \partial \Omega .
\end{aligned}
$$

Suppose $\Omega$ is convex and contained in the unit ball $B_{1}, \varphi \in C^{\alpha}(\bar{\Omega})$ is convex, and $\nu(\Omega) \leq c_{1}$ for a constant $c_{1}$. Then

$$
|u(x)-u(y)| \leq C|x-y|^{\bar{\alpha}} \forall x, y \in \Omega,
$$

where $C$ depends on $n, c_{1}$, and $\|\varphi\|_{C^{\bar{\alpha}}(\bar{\Omega})}$, and $\bar{\alpha}=\min \left(\frac{1}{n}, \alpha\right)$. 
Proof. First consider the case $u \equiv 0$ on $\partial \Omega$. We show that

$$
\left|u\left(x_{0}\right)-u\left(y_{0}\right)\right| \leq C\left|y_{0}-x_{0}\right|^{1 / n}
$$

for $x_{0}, y_{0} \in \bar{\Omega}$. Since $u$ is convex, it suffices to prove the inequality for $x_{0} \in \Omega, y_{0} \in \partial \Omega$. For any point $x_{0} \in \Omega$, by choosing proper coordinates, we assume that $x_{0}=d e_{n}$ and $\Omega \subset\left\{x_{n}>0\right\}$, where $d=\operatorname{dist}\left(x_{0}, \partial \Omega\right)$ and $e_{n}=(0, \cdots, 0,1)$. Then $\Omega \subset \hat{\Omega}=\left\{x \in \mathbb{R}^{n}:\left|x^{\prime}\right|<2,0<x_{n}<4\right\}$. Let $v$ and $w$ be convex functions such that their graphs are convex cones, with vertex at $\left(x_{0}, u\left(x_{0}\right)\right)$ and bases $\partial \Omega$ and $\partial \hat{\Omega}$, respectively. Then $N_{u}(\Omega) \supset$ $N_{v}(\Omega)=N_{v}\left(x_{0}\right) \supset N_{w}\left(x_{0}\right)$. Since $w$ is a convex cone, one easily verifies that $\left|N_{w}\left(x_{0}\right)\right| \geq \frac{C}{d}\left|u\left(x_{0}\right)\right|^{n}$, namely $\left|u\left(x_{0}\right)\right| \leq C[d \nu(\Omega)]^{1 / n}$.

When $u=\varphi$ on $\partial \Omega$ for a convex function $\varphi \in C^{\alpha}(\bar{\Omega})$, we let $u_{0}$ be a solution of $\operatorname{det} D^{2} u=\nu$ in $\Omega$ which vanishes on $\partial \Omega$. Then $u_{0}+\varphi$ is a sub-barrier and we also obtain (2.9).

COROLlARY 2.1. Let $u$ be a generalized solution to (2.6) which vanishes on $\partial \Omega$. Suppose $\nu(\Omega) \leq b \nu\left(\frac{1}{2} \Omega\right)$ for some constant $b>0$. Let $\ell$ be a line segment in $\Omega$ with two endpoints $z^{\prime}, z^{\prime \prime} \in \partial \Omega$. Let $z$ be a point on $\ell$ such that $u(z) \leq \frac{1}{2} \inf _{\Omega} u$. Then $\left|z^{\prime}-z\right| \geq C\left|z^{\prime \prime}-z^{\prime}\right|$ for some $C>0$ depending only on $n$ and $b$.

Note that the ratio $\frac{\left|z^{\prime}-z\right|}{\left|z^{\prime \prime}-z^{\prime}\right|}$ is invariant under linear transforms. Hence by making a linear transform we may assume that $|\Omega|=1$ and $\Omega$ is normalized. By Theorem 2.1 and the assumption $\nu(\Omega) \leq b \nu\left(\frac{1}{2} \Omega\right)$, we may assume furthermore that inf $u=-1$ and $\nu(\Omega) \leq C$. Hence when $u(z) \leq-\frac{1}{2}$, by Theorem 2.2 we have $\operatorname{dist}(z, \partial \Omega) \geq C_{0}>0$, and so Corollary 2.1 follows.

\subsection{Strict convexity}

We say a measure $\nu$ satisfies the doubling condition if there exists a constant $b>0$ such that for any convex set $\omega \subset \Omega$,

$$
\nu(\omega) \leq b \nu\left(\frac{1}{2} \omega\right)
$$

This condition is invariant under affine transforms. We always assume that $\nu(\Omega)>0$. For $x_{0} \in \Omega, h>0$, denote

$$
S_{h, u}^{0}\left(x_{0}\right)=\{x \in \Omega \mid u(x)<\ell(x)+h\},
$$

where $\ell$ is the supporting function of $u$ at $x_{0}$. Sometimes we drop the subscript $u$ if no confusion arises, and also write $S_{h, u}^{0}\left(x_{0}\right)$ as $S_{h}^{0}$ if $x_{0}$ is the minimum point of $u$. First we consider the strict convexity of solutions.

THEOREM 2.3. (Strict convexity) Let u be a generalized solution to (2.6). Assume that $\nu$ satisfies the doubling condition (2.10). Then for any point $x_{0} \in \Omega$, either the contact set $\mathcal{C}=\{x \in \Omega: u(x)=\ell(x)\}$ is a single point, where $\ell$ is a supporting function of $u$ at $x_{0}$, or $\mathcal{C}$ has no extremal points in $\Omega$. 
Proof. By subtracting the supporting function, we assume that $\mathcal{C}=$ $\{u=0\}, u \geq 0$ in $\Omega$. If the claim is not true, by contradiction we assume $\mathcal{C}$ is not a single point and $z \in \Omega$ is an extremal point of $\mathcal{C}$.

By a transform of coordinates we may assume that $z=(0, \cdots, 0, \delta)$ for a small constant $\delta>0$, and $G_{0}=\mathcal{C} \cap\left\{x_{n} \geq 0\right\}$ is compactly contained in $\Omega$.

Let $G_{\varepsilon}=\left\{x \in \Omega: u(x) \leq \varepsilon x_{n}\right\}$, where $\varepsilon>0$ is a constant. Denote $w(x)=u(x)-\varepsilon x_{n}$. When $\varepsilon$ is sufficiently small, we have $\partial G_{\varepsilon} \subset \Omega$. Hence $w$ vanishes on $\partial G_{\varepsilon}$. Let $z^{\prime} \in G_{\varepsilon}$ such that the three points $0, z$ and $z^{\prime}$ lie on a straight line. Observing that $G_{\varepsilon}$ shrinks to the set $G_{0}$ as $\varepsilon \rightarrow 0$, we have $\left|z^{\prime}-z\right|=o\left(\left|z^{\prime}\right|\right)$ and moreover, $\inf _{G_{\varepsilon}} w \geq 2 w(z)=-2 \varepsilon \delta$. Applying Corollary 2.1 to $w$ we reach a contradiction.

Next we rule out the possibility that extremal points of $\mathcal{C}$ lie on the boundary $\partial \Omega$ under some appropriate boundary assumptions.

THEOREM 2.4. Let $u$ be a generalized solution to (2.6) in a convex domain $\Omega$. Assume that $\nu$ satisfies the doubling condition (2.10). Suppose that either

(i) $u=0$ on $\partial \Omega$; or

(ii) $u=\varphi$ on $\partial \Omega$ for some $\varphi \in C^{1, \alpha}$ and $\partial \Omega \in C^{1, \alpha}$ with $\alpha>1-\frac{2}{n}$, and $\nu \geq c_{0}$ for a positive constant $c_{0}$.

Then $u$ is strictly convex in $\Omega$.

The exponent $\alpha>1-\frac{2}{n}$ in (ii) is optimal, as shown by Pogorelov's example

$$
u(x)=\left(1+x_{n}^{2}\right)\left|x^{\prime}\right|^{2\left(1-\frac{1}{n}\right)} .
$$

The function satisfies the equation

$$
\operatorname{det} D^{2} u=2 \beta^{n-1}\left(1+x_{n}^{2}\right)^{n-2}\left(\beta-1-(\beta+1) x_{n}^{2}\right),
$$

where $\beta=2-2 / n$. The right hand side is an analytic positive function when $\left|x_{n}\right|<\frac{\beta-1}{\beta+1}$.

Proof of Theorem 2.4. Case (i): For any point $x_{0} \in \Omega$, let $\ell$ be a supporting function of $u$ at $x_{0}$. If $u$ is not strictly convex at $x_{0}$, by Theorem 2.3 all extremal points of the contact set $\mathcal{C}=\{u=\ell\}$ lie on the boundary $\partial \Omega$. By convexity $u=0$ in $\Omega$, which is a contradiction to $\nu(\Omega)>0$.

Case (ii): For any point $x_{0} \in \Omega$, by subtracting a hyperplane we assume that $u \geq 0$ in $\Omega$ and $u\left(x_{0}\right)=0$. Let $h_{0}=\sup \left\{h \mid S_{h}^{0} \Subset \Omega\right\}$ and let $h=h_{0}+\delta$, where the constant $\delta>0$ will be determined below. Choose a point $x_{b} \in \partial \Omega \cap \partial S_{h_{0}}^{0}$. By a linear transform of the coordinates we assume that $x_{0}=0$ and $x_{b}=(0, \cdots, 0,1)$. By the conditions $\varphi \in C^{1+\alpha}$ ad $\partial \Omega \in C^{1+\alpha}$, we have $u=\varphi \leq h$ on $\partial \Omega \cap B_{r}\left(x_{b}\right)$, where $r=\delta^{1 /(1+\alpha)}$. By the convexity of $u$, we have $u \leq h$ in the cone $\mathcal{C}=\left\{x \in \Omega: \quad x_{n} \geq \frac{1}{r}\left|x^{\prime}\right|\right\}$. 
Let $v$ be the solution to $\operatorname{det} D^{2} v=\nu$ in $S_{h}^{0}$ and $v=h$ on $\partial S_{h}^{0}$. By the first estimate in (2.7), we have

$$
\inf _{S_{h}^{0}}(v-h) \leq-C|\mathcal{C}|^{2 / n} \leq-C \delta^{\frac{2(n-1)}{n(1+\alpha)}} .
$$

By the comparison principle, $u \leq v$ in $S_{h}^{0}$. Hence

$$
-h_{0}-\delta=-h=\inf _{S_{h}^{0}}(u-h) \leq-C \delta^{\frac{2(n-1)}{n(1+\alpha)}} .
$$

When $\alpha>1-\frac{2}{n}, \frac{2(n-1)}{n(1+\alpha)}<1$. Hence when $\delta>0$ is small, we obtain

$$
h_{0} \geq C \delta^{\frac{2(n-1)}{n(1+\alpha)}} .
$$

This inequality implies not only the strict convexity of $u$ but also give an estimate for the modulus of convexity for $u$.

REMARK 2.1. Combining the strict convexity with a normalisation argument one can obtain the following estimate, which is dual to the $C^{1, \alpha}$ estimate in $\S 2.5$.

Corollary 2.2. [33] Under the hypotheses of Theorem 2.4, there exists $\beta>0$, depending on $n$ and $b$, such that for any $x_{0} \in \Omega$,

$$
u(x) \geq C\left|x-x_{0}\right|^{1+\beta}+\ell_{x_{0}}(x),
$$

where $\ell_{x_{0}}$ is a support function of $u$ at $x_{0}$, and $C>0$ is a constant depending on $n, b, \Omega, \nu$, and $\operatorname{dist}(x, \partial \Omega)$.

Proof. The proof was given in $[\mathbf{3 3}]$. We include it here for completeness. By subtracting a linear function, we assume $x_{0}=0, u(0)=0, u \geq 0$ in $\Omega$.

By the strict convexity, $S_{h}^{0}$ is compactly contained in $\Omega$ if $h>0$ is small. Make a linear transform $y=T x$ and $v(y)=u(x) / h$ such that $B_{1 / n} \subset T\left(S_{h}^{0}\right) \subset B_{1}$. When $h \leq C_{0}, v=1$ on $T\left(\partial S_{h}^{0}\right)$. The doubling condition (2.10) and the uniform estimate (Theorem 2.1) implies that $\nu\left[T\left(S_{h}^{0}\right)\right] \leq C_{2}$. Hence by the Hölder continuity of $v$ (Theorem 2.2), we have $\operatorname{dist}\left(T\left(S_{h / 2}^{0}\right), T\left(\partial S_{h}^{0}\right)\right) \geq C_{3}$, where $C_{2}, C_{3}$ depends only on $n, b$. Changing back we obtain

$$
u(\theta x) \geq \frac{1}{2} u(x)
$$

for any $x \in \partial S_{h}^{0}$, where $\theta=1-\frac{1}{2} C_{3}$. As $h$ is any small constant, it follows that for any $x$ near the origin,

$$
u(x) \geq 2^{-k} u\left(\theta^{-k} x\right)
$$

provided $\theta^{-k} x \in \Omega$. Hence we obtain (2.12) with $\beta$ given by $\theta^{1+\beta}=1 / 2$.

\subsection{Strict convexity in dimension two}

In dimension two, we have the following stronger result. 
THEOREM 2.5. Let $u$ be a convex function in $B_{1}(0) \subset \mathbb{R}^{2}$ satisfying $\operatorname{det} D^{2} u \geq c_{0}>0$, where $c_{0}$ is a positive constant. Then $u$ is strictly convex in $\Omega$.

Proof. By subtracting a linear function we may suppose that $u(1,0)=$ $u(-1,0)=0$, and $\inf _{x \in \partial B_{1}}[u(x)+u(-x)]$ is attained at $x=(1,0)$. We need only to show that $u(0) \leq-C$.

By convexity we have $0 \geq u\left(x_{1}, 0\right) \geq-2|u(0)|$ for $x_{1} \in(-1,1)$ and for any $x \in B_{3 / 4}$,

$$
\begin{aligned}
& u\left(x_{1}, x_{2}\right) \geq u\left(x_{1}, 0\right)-C\left|x_{2}\right| \geq-2|u(0)|-C\left|x_{2}\right|, \\
& u\left(x_{1}, x_{2}\right) \leq u\left(x_{1}, 0\right)+C\left|x_{2}\right| \leq C\left|x_{2}\right| .
\end{aligned}
$$

It follows that for any $x \in\left(-\frac{1}{4}, \frac{1}{4}\right) \times\left(-\frac{1}{4}, \frac{1}{4}\right)$,

$$
\partial_{x_{1}} u(x) \leq 8\left(u\left(\frac{1}{2}, x_{2}\right)-u\left(x_{1}, x_{2}\right)\right) \leq 16\left(|u(0)|+C\left|x_{2}\right|\right) .
$$

Similarly we have $\partial_{x_{1}} u(x) \geq-16\left(|u(0)|+C\left|x_{2}\right|\right)$.

Approximating by smooth functions, we may assume that $u$ is smooth. Hence we have $u_{11} u_{22} \geq c_{0}$. Hence

$$
\int_{-1 / 4}^{1 / 4} \frac{1}{u_{22}(x)} d x_{1} \leq c_{0}^{-1} \int_{-1 / 4}^{1 / 4} u_{11} d x_{1} \leq \frac{32}{c_{0}}\left(|u(0)|+C\left|x_{2}\right|\right) .
$$

We obtain

$$
\int_{-1 / 4}^{1 / 4} u_{22}(x) d x_{1} \geq \frac{1}{4}\left(\int_{-1 / 4}^{1 / 4} \frac{1}{u_{22}(x)} d x_{1}\right)^{-1} \geq \frac{c_{0}}{128}\left(|u(0)|+C\left|x_{2}\right|\right)^{-1} .
$$

It follows that

$$
\begin{aligned}
C & \geq \int_{-1 / 4}^{1 / 4}\left[\int_{0}^{1 / 4} u_{22}(x) d x_{2}\right] d x_{1} \\
& =\int_{0}^{1 / 4}\left[\int_{-1 / 4}^{1 / 4} u_{22}(x) d x_{1}\right] d x_{2} \\
& \geq \frac{c_{0}}{128} \int_{0}^{1 / 4}\left(|u(0)|+C\left|x_{2}\right|\right)^{-1} d x_{2} .
\end{aligned}
$$

Hence $u(0) \leq-\epsilon_{0}$ for some $\epsilon_{0}>0$ depending only on $c_{0}$ and the gradient of $u$ in $B_{1}$.

The above strict convexity was due to Aleksandrov, but the above proof was first given in [33].

\section{$2.5 C^{1, \alpha}$ regularity}

First we note that by the Legendre transform, if $u$ is strictly convex at 0 , then $u^{*}$ is $C^{1}$ smooth at any point in $N_{u}(0)$. Moreover, if $u$ satisfies (2.12), then $u^{*}$ is $C^{1, \alpha}$ with $\alpha=1 / \beta$ (see Lemma 3.1 in $[\mathbf{2 3}]$ ). 
Theorem 2.6. Let $u, \Omega$ and $\nu$ be the same as in Theorem 2.4. Then there exists $\alpha \in(0,1]$, depending on $n, b$ such that for any $x_{0} \in \Omega$,

$$
u(x) \leq C\left|x-x_{0}\right|^{1+\alpha}+\ell_{x_{0}}(x),
$$

where $\ell_{x_{0}}$ is a support function of $u$ at $x_{0}, C$ is a constant depending on $n, b, \Omega, \nu$, and $\operatorname{dist}\left(x_{0}, \partial \Omega\right)$.

Proof. By subtracting a linear function, we assume $x_{0}=0, u(0)=0$, $u \geq 0$ in $\Omega$. By the strict convexity of $u$, the set $S_{h}^{0}=\{u<h\} \Subset \Omega$ if $h>0$ is small. Suppose there exists $\sigma>0$ depending only on $n$ and $b$ such that for any small $h>0$ and any $x \in \partial S_{h}^{0}$,

$$
u\left(\frac{1}{2} x\right)<\frac{1-\sigma}{2} u(x)
$$

Define $\alpha$ by $1-\sigma=2^{-\alpha}$. Then for any $x \in \partial S_{h}^{0}$ and any $t \in\left(\frac{1}{2^{k+1}}, \frac{1}{2^{k}}\right)$,

$$
u(t x) \leq 2^{-k}(1-\sigma)^{k} u(x)=\left[2^{-k}\right]^{1+\alpha} u(x) \leq 2 t^{1+\alpha} u(x) .
$$

Hence $u \in C^{1, \alpha}$.

Inequality (2.15) follows from (2.13) with $\sigma=\frac{1-\theta}{5}$. Indeed, consider the convex function $g(t)=u(t x), t \in[-1,1]$. Replacing $g$ by $g / g(1)$, we may assume that $g(1)=1$. Let $\psi(t)=g\left(t+\frac{1}{2}\right)-g^{\prime}\left(\frac{1}{2}\right) t-g\left(\frac{1}{2}\right)$. Then by the convexity of $g, \psi(0)=0, \psi \geq 0$. If $g\left(\frac{1}{2}\right)>\frac{1-\epsilon}{2}$, by convexity we have $1+\epsilon \geq g^{\prime}\left(\frac{1}{2}\right) \geq 1-\epsilon$ and $\psi\left(-\frac{1}{2}\right) \leq \epsilon$. Applying (2.13) to $\psi$, we have $\psi\left(-\frac{1}{2} \theta^{-1}\right) \leq 2 \psi\left(-\frac{1}{2}\right) \leq 2 \epsilon$. Hence $g\left(-\frac{1}{2} \theta^{-1}+\frac{1}{2}\right)<0$ when $\epsilon<\frac{1-\theta}{5}$, we reach a contradiction as $u \geq 0$.

The $C^{1, \alpha}$ regularity is not true if the measure $\nu$ does not satisfies the doubling condition. See examples in [35]

\section{3. $C^{2, \alpha}$ estimate}

3.1. Statement of the result. In order to state the main results, let us first introduce some notations and terminologies. Let $u$ be a convex function in $\Omega$. For any point $y \in \Omega$ and constant $h>0$, denote

$$
S_{h, u}^{0}(y)=\left\{x \in \Omega: u(x)<\ell_{y}(x)+h\right\},
$$

where $\ell_{y}$ is a support function of $u$ at $y$. The modulus of convexity of $u$ is a nonnegative function given by

$$
\rho_{u}(r)=\inf _{y \in \Omega} \rho_{u, y}(r), \quad r>0,
$$

where

$$
\rho_{u, y}(r)=\sup \left\{h \geq 0: S_{h, u}^{0}(y) \subset B_{r}(y)\right\}
$$

if there exists $h \geq 0$ such that $S_{h, u}^{0}(y) \subset B_{r}(y)$; or $\rho_{u, y}(r)=0$ otherwise. A convex function $u$ is strictly convex if $\rho_{u}(r)>0$ for all $r>0$.

Denote

$$
\omega_{f}(r)=\sup \{|f(x)-f(y)|:|x-y|<r\} .
$$


For any $r_{1}, r_{2}>0$, apparently

$$
\omega_{f}\left(r_{1}\right)+\omega_{f}\left(r_{2}\right) \geq \omega_{f}\left(r_{1}+r_{2}\right) .
$$

In particular, we have

$$
\omega_{f}(C r) \leq C \omega_{f}(r) .
$$

We say $f$ is Dini continuous if

$$
\int_{0}^{1} \frac{\omega_{f}(r)}{r} d r<\infty
$$

TheOrem 3.1. Let $u \in C^{2}$ be a strictly convex solution of (1.1). Assume that $f$ satisfies

$$
C_{1} \leq f \leq C_{2}
$$

for some constants $C_{1}, C_{2}>0$. Then for all $x, y \in \Omega^{\prime} \Subset \Omega$, we have the estimate

$$
\left|D^{2} u(x)-D^{2} u(y)\right| \leq C\left[d+\int_{0}^{d} \frac{\omega_{f}(r)}{r}+d \int_{d}^{1} \frac{\omega_{f}(r)}{r^{2}}\right],
$$

where $d=|x-y|, C>0$ depends only on $n, \rho_{u}, \Omega^{\prime}$ and $C_{1}, C_{2}$. It follows that

(i) If $f$ is Dini continuous, then (3.2) gives an estimate for the modulus of continuity of $D^{2} u$.

(ii) If $f \in C^{\alpha}(\Omega)$ and $\alpha \in(0,1)$, then

$$
\|u\|_{C^{2, \alpha}\left(\Omega^{\prime}\right)} \leq C\left[1+\frac{\|f\|_{C^{\alpha}(\Omega)}}{\alpha(1-\alpha)}\right] .
$$

(iii) If $f \in C^{0,1}(\Omega)$, then

$$
\left|D^{2} u(x)-D^{2} u(y)\right| \leq C d\left[1+\|f\|_{C^{0,1}}|\log d|\right] .
$$

Note that the constant $C$ depends in the modulus of convexity of $u$, which implies that $C$ also depends on $\operatorname{osc}_{\Omega} u$. The $C^{2}$ estimate in (i) and the $C^{2, \alpha}$ estimate in (ii) were proved in [19] and [4], respectively. Here, the estimate of the form (3.2) is from the paper [19]. A similar type estimate for the Laplacian and heat equations was previously obtained in [37]. The proof is based on a perturbation argument, which was inspired by the original idea of Caffarelli [4].

3.2. Proof of Theorem 3.1. Let us collect some relevant facts and results $[\mathbf{1 9}]$ which will be needed in the subsequent proof.

Let $\Omega$ be a bounded convex domain and let $T$ be a unimodular linear transformation such that $T(\Omega)$ is normalized. Choose an appropriate coordinate system such that the minimum ellipsoid of $\Omega$ is given by $E=\left\{\sum_{i=1}^{n} \frac{x_{i}^{2}}{a_{i}^{2}}<1\right\}$ with $a_{1} \geq \cdots \geq a_{n}$. Then

$$
T(x)=\left(\lambda_{1} x_{1}, \cdots, \lambda_{n} x_{n}\right)
$$


with

$$
\lambda_{i}=\frac{1}{a_{i}}\left(a_{1} \cdots a_{n}\right)^{1 / n}, \quad i=1,2, \cdots, n .
$$

Note that $\lambda_{1}$ and $\lambda_{n}$ are the least and largest eigenvalues of $T$. For convenience we say $\Omega$ has a good shape if

$$
\lambda_{n} \leq c^{*} \lambda_{1}
$$

for some constant $c^{*}$ under control. If $\Omega$ has a good shape, then there exist two concentric balls $B_{r}$ and $B_{R}$ with $R \leq n c^{*} r$ such that $B_{r} \subset \Omega \subset B_{R}$.

LEMMA 3.1. Let $u$ be a convex solution of $\operatorname{det} D^{2} u=1$ in $\Omega$ and $u=0$ on $\partial \Omega$. Suppose that $u$ attains its minimum at the origin and $D^{2} u(0)$ is the unit matrix (or uniformly bounded), then the domain $\Omega$ is of good shape.

In the following, we call the set $S_{h, u}^{0}(y)$ in (3.1) the sub-level set of $u$ at $y$ with height $h$ and denote $S_{h, u}(y)=\partial S_{h, u}^{0}(y)$ its boundary. When no confusion arises we will sometimes drop the subscript $u$, and when $y$ is the minimum point of $u$, we will simply write the sub-level set as $S_{h}^{0}$.

Lemma 3.2. Let $u_{i}, i=1,2$, be two convex solutions of $\operatorname{det} D^{2} u=1$ in $S_{1, u_{1}}^{0}$. Suppose that $\left\|u_{i}\right\|_{C^{4}} \leq C_{0},\left|u_{1}-u_{2}\right| \leq \delta$ for some small constant $\delta>0$, and $B_{1 / n} \subset S_{1, u_{1}}^{0} \subset B_{1}$ is normalized. Then we have, for $1 \leq k \leq 3$,

$$
\left|D^{k}\left(u_{1}-u_{2}\right)\right| \leq C \delta \quad \text { in } \quad S_{3 / 4, u_{1}}^{0},
$$

where $C$ depends only on $n$ and $C_{0}$.

We are now ready to present the proof of Theorem 3.1.

Proof of Theorem 3.1. By subtracting a linear function we suppose

$$
u(0)=0, \quad D u(0)=0,
$$

so that the origin is the minimum point of $u$. Choose $h>0$ small such that the sub-level set $S_{h}^{0}$ is compactly contained in $\Omega$. Let $T_{h}$ be a unimodular linear transform such that $T_{h}\left(S_{h}^{0}\right)$ is normalized. Hence by making the change $x \rightarrow T_{h} x / \sqrt{h}$ and $u \rightarrow u / h$, we may suppose $h=1, S_{1}^{0}$ is normalized, and

$$
\int_{0}^{1} \frac{\omega(r)}{r} \leq \epsilon
$$

where $\omega(r)=\omega_{f}(r), \epsilon$ can be as small as we want, provided $h$ is sufficiently small.

Let $u_{k}, k=0,1, \cdots$, be the solution of

$$
\begin{aligned}
\operatorname{det} D^{2} u_{k} & =f(0) \quad \text { in } S_{4^{-k}, u}^{0}, \\
u_{k} & =u\left(=4^{-k}\right) \text { on } \partial S_{4^{-k}, u}^{0} .
\end{aligned}
$$

Denote

$$
\begin{aligned}
\nu(t) & =\sup _{z \in \Omega}\left\{|f(x)-f(y)|: x, y \in S_{t^{2}, u}^{0}(z)\right\} \\
\nu_{k} & =\nu\left(2^{-k}\right)
\end{aligned}
$$


which is invariant under unimodular linear transformation of $x$. If $S_{t^{2}, u}^{0}$ has good shape, then we have $\nu(t) \leq \omega(C t) \leq C \omega(t)$.

Since $S_{1, u}^{0}$ has a good shape, by the classical solvability of Dirichlet problems $[\mathbf{1 5}],\left\|u_{0}\right\|_{C^{4}\left(S_{3 / 4, u}^{0}\right)} \leq C$. Note that

$$
\operatorname{det} D^{2}\left(1-C \nu_{0}\right) u \leq \operatorname{det} D^{2} u_{0} \leq \operatorname{det} D^{2}\left(1+C \nu_{0}\right) u \quad \text { in } S_{1, u}^{0},
$$

for some constant $C$, and $u=u_{0}=1$ on the boundary. By the comparison principle, we have

$$
\left(1+C \nu_{0}\right)(u-1) \leq u_{0}-1 \leq\left(1-C \nu_{0}\right)(u-1) .
$$

It follows that $\left|u-u_{0}\right| \leq C \nu_{0}$. Similarly we have $\left|u-u_{1}\right| \leq C \nu_{1}$. Hence we obtain $\left|u_{1}-u_{0}\right| \leq C \nu_{0}$. Since $S_{1, u}^{0}$ has a good shape, so does $S_{4^{-1}, u}^{0}$. It follows $\left\|u_{1}\right\|_{C^{4}\left(S_{3 / 16, u_{1}}^{0}\right)} \leq C$. By Lemma 3.2 we obtain

$$
\left|D^{k} u_{0}(x)-D^{k} u_{1}(x)\right| \leq C \nu_{0}
$$

for $x \in S_{4^{-2}, u_{1}}^{0}$, where $1 \leq k \leq 3$. Lemma 3.1 then implies that $S_{4^{-2}, u_{1}}^{0}$ has a good shape.

By induction we assume that $S_{4^{-k-1}, u}^{0}$ has a good shape with the constant $c^{*}$ in (3.5) independent of $k$. Applying the same argument to $\hat{u}_{0}:=4^{k} u_{k}\left(\frac{x}{2^{k}}\right)$ and $\hat{u}_{1}:=4^{k} u_{k+1}\left(\frac{x}{2^{k}}\right)$, we obtain, for $x \in S_{4^{-k-2}, u_{k+1}}^{0}$,

$$
\begin{aligned}
\left|D u_{k}(x)-D u_{k+1}(x)\right| & \leq C 2^{-k} \nu_{k} \\
\left|D^{2} u_{k}(x)-D^{2} u_{k+1}(x)\right| & \leq C \nu_{k} \\
\left|D^{3} u_{k}(x)-D^{3} u_{k+1}(x)\right| & \leq C 2^{k} \nu_{k}
\end{aligned}
$$

where $2^{k}$ in (3.9) is the scaling constant. Hence

$$
\begin{aligned}
\left|D^{2} u_{0}(x)-D^{2} u_{k+1}(x)\right| & \leq C \sum_{i=0}^{k} \nu_{i} \\
& \leq C \int_{2^{-k}}^{1} \frac{\omega(r)}{r} d r \leq C \epsilon
\end{aligned}
$$

for $x \in S_{4^{-k-2}, u_{k+1}}^{0}$, where $C>0$ is independent of $k$. Therefore, $D^{2} u(0)$ is uniformly bounded. This implies that equation (1.1) is uniformly elliptic and Theorem 3.1 follows from [37].

REMARK 3.1. Instead of applying the estimate for uniformly elliptic equation in [37], one can directly prove the estimate (3.2) as in [19]. For any given point $z$ near the origin,

$$
\begin{aligned}
& \left|D^{2} u(z)-D^{2} u(0)\right| \leq I_{1}+I_{2}+I_{3}=: \\
& \left|D^{2} u_{k}(z)-D^{2} u_{k}(0)\right|+\left|D^{2} u_{k}(0)-D^{2} u(0)\right|+\left|D^{2} u(z)-D^{2} u_{k}(z)\right| .
\end{aligned}
$$


Choose $k \geq 1$ such that $4^{-k-4} \leq u(z) \leq 4^{-k-3}$. Then by (3.8),

$$
I_{2} \leq C \sum_{j=k}^{\infty} \nu_{j} \leq C \int_{0}^{|z|} \frac{\omega(r)}{r} .
$$

To estimate $I_{3}$, we consider the sub-level sets $S_{4^{-j}, u}^{0}(z)$ of $u$ at $z$. Similarly to $(3.11)$ we have

$$
I_{3}=\left|D^{2} u(z)-D^{2} u_{k}(z)\right| \leq C \int_{0}^{|z|} \frac{\omega(r)}{r} .
$$

To estimate $I_{1}$, denote $h_{j}=u_{j}-u_{j-1}$. By (3.8),

$$
\left|D^{2} h_{j}(z)-D^{2} h_{j}(0)\right| \leq C 2^{j} \nu_{j}|z| \text {. }
$$

Hence

$$
\begin{aligned}
I_{1} & \leq\left|D^{2} u_{k-1}(z)-D^{2} u_{k-1}(0)\right|+\left|D^{2} h_{k}(z)-D^{2} h_{k}(0)\right| \\
& \leq\left|D^{2} u_{0}(z)-D^{2} u_{0}(0)\right|+\sum_{j=1}^{k}\left|D^{2} h_{j}(z)-D^{2} h_{j}(0)\right| \\
& \leq C|z|\left(1+\sum_{j=1}^{k} 2^{j} \nu_{j}\right) \\
& \leq C|z|\left(1+\int_{|z|}^{1} \frac{\omega(r)}{r^{2}}\right) .
\end{aligned}
$$

Hence we obtain (3.2). Note that (3.3) and (3.4) follows readily from (3.2).

\section{4. $W^{2, p}$ estimate}

4.1. Statement of the result. In this section we prove the $W^{2, p}$ estimate by following Caffarelli's approach [4] with some simplification. The basic observation is that when $f$ is continuous, the sub-level set $S_{h}^{0}$ is a small perturbation of a ball (after normalization), and the solution is a small perturbation of a quadratic function. It implies that in a sufficiently dense set, the second derivative is close to that of the quadratic function.

The main result in this section is the following

THEOREM 4.1. Let $u$ be a strictly convex solution of

$$
\operatorname{det} D^{2} u=f \quad \text { in } \Omega \text {. }
$$

Then for any $p \geq 1$, there exists $\epsilon=\epsilon(p)>0$ such that when

$$
1 \leq f \leq 1+\epsilon
$$

we have $D^{2} u \in L^{p}\left(\Omega^{\prime}\right)$ and the estimate

$$
\|u\|_{W^{2, p}\left(\Omega^{\prime}\right)} \leq C,
$$

where $\Omega^{\prime} \Subset \Omega, C$ depends on $n, p, \epsilon, \Omega, \Omega^{\prime}$, and the modulus of convexity of $u$. 
Corollary 4.1. Let $u$ be a strictly convex solution of (4.1). If $0<$ $C_{1} \leq f \leq C_{2}$ and $f \in C^{0}(\Omega)$, then $D^{2} u \in L^{p}\left(\Omega^{\prime}\right)$ for any $1 \leq p<\infty$, and we have the estimate (4.3), where $C$ depends on $n, p, \Omega, \Omega^{\prime}$, and the modulus of convexity of $u$.

Note that the condition (4.2) cannot be removed. There exists a function $f$ satisfying $C^{-1} \leq f \leq C$ such that $u \notin W^{2, p}$ for large $p[35]$.

4.2. Proof of Theorem 4.1. We recall a Calderón-Zygmund type decomposition from $[\mathbf{4}, \mathbf{1 6}]$ which is needed in our proof.

Covering Lemma: Let $A, B \Subset \Omega$ be two subsets of $\Omega$. Let $\mathcal{F}=\left\{S_{h_{x}, u}^{0}(x)\right.$ : $x \in B\}$ be a family of sub-level sets such that $A \subset \cup_{x \in B} S_{h_{x}, u}^{0}(x)$. Then there exists a sequence $\left\{S_{i}, i=1,2, \cdots\right\}$ satisfying that $A \subset \cup S_{i}$, where $S_{i}=S_{h_{x_{i}}, u}^{0}\left(x_{i}\right)$, and there exists a constant $K$ such that any two sub-level sets in $\left\{S_{h_{i} / K}^{0}\left(x_{i}\right)\right\}$ are disjoint.

Moreover, we assume the following additional assumption, whose proof is postponed to the next subsection.

Assumption $H$ : For any $N>10$ and $\delta>0$, there exists $\epsilon>0$ such that for any convex solution $u$ of (4.1) satisfying $u(0)=0, u \geq 0$ and $S_{1}^{0}$ is normalized, we have $|D| \geq(1-\delta)\left|S_{1}^{0}\right|$ where

$$
\begin{aligned}
D & =\left\{y \in S_{1}^{0}: u(x) \geq \ell_{y}(x)+\frac{1}{N}|x-y|^{2} \quad \forall x \in S_{1}^{0}(y)\right\} \\
& =\left\{y \in S_{1}^{0}: S_{h}^{0}(y) \subset B_{\sqrt{N h}}(y) \quad \forall h \in(0,1)\right\},
\end{aligned}
$$

where $\ell_{y}$ is the tangent plane of $u$ at $y$.

Now, let $u$ be a convex solution of (4.1) defined in $B_{M}(0)$ for a large $M>1$, with the properties that $u(0)=0, u \geq 0$, and the set $S_{1}^{0}$ is normalized. Denote

$$
\begin{aligned}
D_{k} & =\left\{x \in S_{1, u}^{0}: D^{2} u(x)>2 N^{-k} I\right\} \\
& =\left\{x \in S_{1, u}^{0}: \exists h_{x}>0 \text { such that } S_{h}^{0}(x) \subset B_{\sqrt{N^{k} h}}(x) \quad \forall h<h_{x}\right\}, \\
A_{k} & =S_{1, u}^{0}-D_{k}, \quad k=1,2, \cdots,
\end{aligned}
$$

where $h_{x}$ depends on $x$. In the following lemma we show fast polynomial decay of the measure of $A_{k}$, which implies an $L^{p}$ estimate of $D^{2} u$.

Lemma 4.1. For any $q<\infty$, there exists $\epsilon=\epsilon(q)>0$ such that if $|f-1|<\epsilon$, then

$$
\left|A_{k} \cap B_{r_{k}}(0)\right| \leq N^{-q k},
$$

where $r_{1}=\frac{1}{n}, r_{k+1}$ is given by

$$
r_{k+1}=r_{k}-N^{-\frac{k}{2(\beta-2)}} .
$$

where $\beta$ is the constant in (4.11) below. 
Proof. We divide the proof into three steps.

Step 1: For $y \in A_{k}$, let

$$
\hat{h}_{y}=\inf \left\{h>0: S_{h}^{0}(y) \subset B_{\sqrt{N^{k} h}}(y)\right\} .
$$

By our definition of $A_{k}$, we have $\hat{h}_{y}>0$ for any $y \in A_{k}$.

Let

$$
\bar{u}(x)=\frac{1}{\hat{h}_{y}}\left[u-\ell_{y}\right]\left(T_{y}(x)\right),
$$

where $T_{y}$ is a linear transform such that $\bar{u}$ satisfies the conditions in Assumption H.

By Assumption $\mathrm{H}$, there exists a set $D=D_{\bar{u}} \subset S_{1, \bar{u}}^{0}$ satisfying $|D| \geq$ $(1-\delta)\left|S_{1, \bar{u}}^{0}\right|$ and for any $z \in D$,

$$
\bar{u}(x) \geq \bar{\ell}_{z}(x)+\frac{1}{N}|x-z|^{2} \quad \forall x \in S_{1, \bar{u}}^{0}(z),
$$

where $\bar{\ell}_{z}$ is the tangent plane of $\bar{u}$ at $z$.

Note that if $x_{0} \in D_{k-1}$, then after normalization, the sub-level set $S_{h, u}^{0}\left(x_{0}\right)$ cannot have a good shape, in the sense that

$$
S_{h, u}^{0}\left(x_{0}\right) \not \subset B_{\sqrt{N h}}\left(x_{0}\right)
$$

when $h$ is sufficiently small.

Scaling back to $u$ in the set $S_{\hat{h}_{y}, u}^{0}(y)$, we see that there is a set $E_{y} \subset$ $S_{\hat{h}_{y}, u}^{0}(y)$ with

$$
\frac{\left|S_{\hat{h}_{y}, u}^{0}(y)-E_{y}\right|}{\left|S_{\hat{h}_{y}, u}^{0}(y)\right|} \leq \delta
$$

and for any $x \in E_{y}$, there exists $h_{x}>0$ such that

$$
S_{h, u}^{0}(x) \subset B_{\sqrt{N^{k+1} h}}(x) \quad \forall h \leq h_{x} .
$$

Hence

$$
E_{y} \subset D_{k+1}, \quad \text { i.e. } E_{y} \cap A_{k+1}=\emptyset .
$$

Moreover by (4.7), if $x \in E_{y}$, then $x \notin D_{k-1}$. Hence we also have

$$
E_{y} \subset A_{k-1} \text {. }
$$

Step 2: Let $y$ and $\hat{h}_{y}$ be as in (4.6). Subtract a linear function such that $u(y)=0, D u(y)=0$. By the strict convexity, there exists a constant $\beta>2$ such that $u(x) \geq C|x|^{\beta}$ near $x=0$. Hence

$$
\hat{h}_{y} \geq C\left[N^{k} \hat{h}_{y}\right]^{\beta / 2},
$$

that is,

$$
\hat{h}_{y} \leq C^{\frac{-2}{\beta-2}} N^{-\frac{k \beta}{\beta-2}} .
$$


We obtain

$$
N^{k} \hat{h}_{y} \leq C^{\frac{-2}{\beta-2}} N^{-\frac{2 k}{\beta-2}} \leq N^{-\frac{k}{\beta-2}}
$$

when $N$ is chosen large. That is

$$
\operatorname{diam}\left(S_{\hat{h}_{y}, u}^{0}(y)\right) \leq N^{-\frac{k}{2(\beta-2)}} .
$$

By our choice of $r_{k}$, we see that if $y \in A_{k+1} \cap B_{r_{k+1}}(0)$, then

$$
S_{\hat{h}_{y}, u}^{0}(y) \subset B_{r_{k}}(0) .
$$

We point out that $(4.12)$ can also be derived from the $C^{1, \alpha}$ regularity of generalized solutions $[\mathbf{2 4}]$.

Step 3: The set of all sub-level sets $\left\{S_{\hat{h}_{y} / K, u}^{0}(y), y \in A_{k}\right\}$ is obviously a covering of $A_{k} \cap B_{r_{k}}(0)$, where $\hat{h}_{y}$ is given by (4.6) and $K$ is the constant in the covering lemma. By the covering lemma, there exists a countable set $\left\{y_{i}\right\} \subset A_{k} \cap B_{r_{k}}(0), i=1,2, \cdots$, such that

$(\mathrm{P} 1), A_{k} \cap B_{r_{k}}(0) \subset \bigcup S_{\hat{h}_{y_{i}}, u}^{0}\left(y_{i}\right)$; and

$(\mathrm{P} 2)$, any two sub-level sets in $\left\{S_{\hat{h}_{y_{i}} / K}^{0}\left(y_{i}\right)\right\}$ are disjoint.

Denote $S_{i}=S_{\hat{h}_{y_{i}}, u}^{0}\left(y_{i}\right)$ and $S_{i}^{\prime}=S_{\hat{h}_{y_{i}} / K}^{0}\left(y_{i}\right)$. From (4.7), there exists a set $E_{y_{i}} \subset S_{i}$ such that

$$
\frac{\left|S_{i}-E_{y_{i}}\right|}{\left|S_{i}\right|} \leq \delta
$$

and $E_{y_{i}}$ satisfies (4.9), (4.10).

Since $A_{k+1} \subset A_{k}$, by the covering property (P1) we have

$$
\left|A_{k+1} \cap B_{r_{k+1}}(0)\right| \leq\left|A_{k+1} \cap\left(\cup S_{i}\right)\right| \leq \sum_{i}\left|A_{k+1} \cap S_{i}\right| .
$$

By (4.13) we have

$$
\left|A_{k+1} \cap S_{i}\right| \leq \delta\left|S_{i}\right|
$$

By the convexity of $u$ we have

$$
\left|S_{i}\right| \leq K^{n}\left|S_{i}^{\prime}\right|
$$

By (4.8)

$$
\left|S_{i}^{\prime}-E_{y_{i}}\right| \leq \delta\left|S_{i}\right| \leq K^{n} \delta\left|S_{i}^{\prime}\right| .
$$

Writing $S_{i}^{\prime}=\left(S_{i}^{\prime}-E_{y_{i}}\right) \cup\left(E_{y_{i}} \cap S_{i}^{\prime}\right)$ we then have

$$
\left|S_{i}^{\prime}\right| \leq \frac{1}{1-K^{n} \delta}\left|E_{y_{i}} \cap S_{i}^{\prime}\right| \leq 2\left|\hat{S}_{i}\right|
$$

when $K^{n} \delta<\frac{1}{2}$, where $\hat{S}_{i}=S_{i}^{\prime} \cap E_{y_{i}}$. We therefore obtain

$$
\left|A_{k+1} \cap B_{r_{k+1}}(0)\right| \leq 2 K^{n} \delta \sum\left|\hat{S}_{i}\right| .
$$


By (4.10), $E_{y_{i}} \subset A_{k-1}$, and by (4.12), $S_{i}^{\prime} \subset B_{r_{k-1}}$. Hence $\hat{S}_{i} \subset\left(A_{k-1} \cap B_{r_{k-1}}\right)$ and so

$$
\left|A_{k+1} \cap B_{r_{k+1}}(0)\right| \leq 2 K^{n} \delta \sum\left|\hat{S}_{i} \cap\left(A_{k-1} \cap B_{r_{k-1}}\right)\right| .
$$

By property $(\mathrm{P} 2), \hat{S}_{i}$ are mutually disjoint. Hence

$$
\left|A_{k+1} \cap B_{r_{k+1}}(0)\right| \leq 2 K^{n} \delta\left|\left(\cup \hat{S}_{i}\right) \cap\left(A_{k-1} \cap B_{r_{k-1}}\right)\right| .
$$

But $\cup \hat{S}_{i} \subset A_{k-1} \cap B_{r_{k-1}}$. We finally obtain

$$
\left|A_{k+1} \cap B_{r_{k+1}}(0)\right| \leq 2 K^{n} \delta\left|A_{k-1} \cap B_{r_{k-1}}\right| .
$$

Therefore for any given $q<\infty$, we can choose $\delta$ small enough such that $2 \delta K^{n} \leq N^{-2 q}$. We obtain the desired estimate (4.5).

Theorem 4.1 now follows from Lemma 4.1 easily.

Proof of Theorem 4.1. It suffices to prove Theorem 4.1 on a sublevel set which after proper rescaling satisfies the conditions in Assumption H.

Denote $A_{k}^{\prime}=A_{k}-A_{k+1}$. Then in $A_{k}^{\prime}$, we have $\left|D^{2} u\right| \leq C N^{(n-1) k}$. Hence

$$
\begin{aligned}
\int_{B_{1 / 2 n}(0)}\left|D^{2} u\right|^{p} d x & \leq C \sum N^{(n-1) k p}\left|A_{k}^{\prime}\right| \\
& \leq C \sum N^{(n-1) k p} N^{-k q} .
\end{aligned}
$$

Letting $q>(n-1) p$, we obtain $u \in W^{2, p}\left(\Omega^{\prime}\right)$.

4.3. Proof of Assumption H. Given $u \in C^{0}(\bar{\Omega})$, denote

$$
\Gamma[u]=\sup v,
$$

where the sup is taken in the set of all affine functions $v$ satisfying $v \leq u$ in $\Omega$. Then $\Gamma[u]$ is convex, and is called the convex envelope of $u$ in $\Omega$. Apparently

$$
\Gamma[u] \leq u
$$

LEMma 4.2. Let $u \in C^{2}(\bar{\Omega})$ be a convex solution of (4.1) and $v \in C^{2}(\bar{\Omega})$ be a convex function. Suppose $f<1+\epsilon$. Then for any Borel set $E \Subset \Omega$,

$$
\mu_{\Gamma}(E) \leq \int_{\mathcal{C} \cap E}\left[(1+\epsilon)^{1 / n}-\operatorname{det}^{1 / n} D^{2} v\right]^{n} d x,
$$

where $\Gamma:=\Gamma[u-v], \mu_{\Gamma}$ is the Monge-Ampère measure associated with $\Gamma$ $[4,16,33]$ and $\mathcal{C}=\{x \in \Omega: \Gamma[u-v](x)=(u-v)(x)\}$ is the contact set.

Proof. From the smoothness of $u, v$, we have $\Gamma \in C^{1,1}$ and $\mu_{\Gamma}=$ $\operatorname{det} \partial^{2} \Gamma d x$, see for example Section 2 in [33]. For any point $x \in \mathcal{C}$ where $\Gamma$ is twice differentiable, we have

$$
D^{2} \Gamma(x) \leq D^{2}(u-v)(x) .
$$


By the concavity of $\operatorname{det}^{1 / n}$

$$
\begin{aligned}
\operatorname{det}^{1 / n}\left[D^{2} u-D^{2} v\right] & \leq \operatorname{det}^{1 / n} D^{2} u-\operatorname{det}^{1 / n} D^{2} v \\
& \leq(1+\epsilon)^{1 / n}-\operatorname{det}^{1 / n} D^{2} v
\end{aligned}
$$

Hence at $x$,

$$
\operatorname{det} D^{2} \Gamma \leq\left[(1+\epsilon)^{1 / n}-\operatorname{det}^{1 / n} D^{2} v\right]^{n}
$$

Recall that $\mu_{\Gamma}=0$ in $\Omega-\mathcal{C}[\mathbf{4}, \mathbf{1 6}, \mathbf{3 3}]$. We obtain (4.16).

LEMmA 4.3. Let $u$ be a $C^{2}$ solution of (4.1) such that $S:=S_{2, u}^{0} \Subset \Omega$. Let $w$ be a solution to $\operatorname{det} D^{2} w=1$ in $S$ such that $w=u$ on $\partial S$. Suppose $1<f<1+\epsilon$. Then

$$
\left|\left\{\Gamma\left[u-\frac{1}{2} w\right]=u-\frac{1}{2} w\right\} \cap S\right| \geq(1-C \epsilon)|S|,
$$

where the constant $C>0$ depends only on $n$.

Proof. By subtracting a constant we assume that $u=w=0$ on $\partial S$. By the comparison principle we have $(1+C \epsilon) w \leq u \leq w$. Hence

$$
\left(\frac{1}{2}+C \epsilon\right) w \leq u-\frac{1}{2} w \leq \frac{1}{2} w .
$$

Since $w$ is convex, we have

$$
\left(\frac{1}{2}+C \epsilon\right) w \leq \Gamma \leq \frac{1}{2} w
$$

where $\Gamma=\Gamma\left[u-\frac{1}{2} w\right]$. As $S$ is convex and $u=w=0$, we also have $\Gamma=0$ on $\partial S$. Hence

$$
\left(\frac{1}{2}+C \epsilon\right) D w(S) \supset D \Gamma(S) \supset \frac{1}{2} D w(S) .
$$

From the second inclusion we have

$$
\mu_{\Gamma}(S) \geq \mu_{\frac{1}{2} w}(S)
$$

On the other hand, by Lemma 4.2,

$$
\begin{aligned}
\mu_{\Gamma}(S) & \leq \int_{S \cap \mathcal{C}}\left[(1+\epsilon)^{1 / n}-\operatorname{det}^{1 / n} D^{2}\left(\frac{1}{2} w\right)\right]^{n} d x \\
& =\int_{S \cap \mathcal{C}}\left[(1+\epsilon)^{1 / n}-\frac{1}{2}\right]^{n} d x \\
& \leq\left(2^{-n}+C \epsilon\right)|S \cap \mathcal{C}|
\end{aligned}
$$

where $\mathcal{C}=\left\{\Gamma=u-\frac{1}{2} w\right\}$, and $C$ depends only on $n$. Since $\operatorname{det} D^{2} w=1$, we have

It follows that

$$
\mu_{\frac{1}{2} w}(S)=2^{-n}|S|
$$

$$
|S| \leq(1+C \epsilon)|S \cap \mathcal{C}|
$$

Note that $\mathcal{C} \subset S$. Hence $|\mathcal{C}| \geq(1-C \epsilon)|S|$. 
Proof of Assumption H. Let $u, w, \mathcal{C}$ be as in Lemma 4.3. If $S_{1, u}^{0}$ is normalized and $u$ is a solution of (4.1) in $\Omega$ which encloses $B_{M}(0)$ for some large $M$, then $S_{2, u}^{0}$ is compactly contained in $B_{M}(0)$ and $\|w\|_{C^{2}\left(S_{1, u}^{0}\right)} \leq C$ for some $C>0$ depending only on $n$.

For any point $y \in \mathcal{C}$, let $\ell_{y}$ be the support function of $\Gamma$ at $y$. Then

$$
\left\{\begin{array}{l}
\left(u-\frac{1}{2} w\right)(x) \geq \ell(x) \text { in } S_{2, u}^{0}, \\
\left(u-\frac{1}{2} w\right)(y)=\ell(y) .
\end{array}\right.
$$

That is

$$
\left\{\begin{array}{l}
u(x) \geq \ell(x)+\frac{1}{2} w(x) \text { in } S_{2, u}^{0} \\
u(y)=\ell(y)+\frac{1}{2} w(y)
\end{array}\right.
$$

Let $D=\mathcal{C} \cap S_{1, u}^{0}$. Since $w$ is smooth and uniformly convex, we obtain (4.4) for some $N>0$ depending only on $\|w\|_{C^{2}\left(S_{1, u}^{0}\right)}$.

Note that in the proof of Theorem 4.1 (§3.2), we used only the affine invariance of the Monge-Ampère equation and the strict convexity of solutions. The condition $1 \leq f \leq 1+\epsilon$ is used in the proof of Assumption H. This condition can be weakened to the following VMO type condition, that is,

$$
\frac{1}{|\omega|} \int_{\omega}|f-1| d x \leq \epsilon
$$

for any convex subset $\omega \subset \Omega$ with nonempty interior (see $[\mathbf{1 8}]$ ).

\section{5. $W^{2,1+\varepsilon}$ estimate}

5.1. Statement of the result. For strictly convex solution $u$ of (1.1), if $f$ solely satisfies

$$
0<C_{1} \leq f \leq C_{2}<\infty \text { in } \Omega
$$

for some constants $C_{1}$ and $C_{2}$, Caffarelli proved [5] that $u \in C_{\mathrm{loc}}^{1, \alpha}(\Omega)$ for some $\alpha \in(0,1)$. For the regularity of the second derivatives of $u$ under the assumption (5.1), counterexamples in [35] show that $u \notin W^{2, p}$ for large $p>1$ depending on the ratio $\frac{C_{2}}{C_{1}}$. By taking $\frac{C_{2}}{C_{1}}$ large enough, $p$ can be chosen as close to 1 as desired. Recently, De Philippis-Figalli-Savin [12] and Schmidt [30] proved $u \in W_{\mathrm{loc}}^{2,1+\varepsilon}$ for some small $\varepsilon$ depending on $n, C_{1}$ and $C_{2}$. The main result can be stated as follows:

THEOREM 5.1. Let $u$ be a strictly convex solution of (1.1), where $f$ satisfies (5.1). Then we have the estimate

$$
\|u\|_{W^{2,1+\varepsilon}\left(\Omega^{\prime}\right)} \leq C
$$

where $\Omega^{\prime} \Subset \Omega, \varepsilon, C>0$ depends on $n, \Omega^{\prime}, C_{1}, C_{2}$ and the modulus of convexity of $u$. 
5.2. Proof of Theorem 5.1. The proof follows from some modifications of the strategy in $\S 3$. We divide the proof into four steps.

1. Let $u$ be a strictly convex solution of (1.1) such that $u(0)=0, u \geq 0$, and $S_{1}^{0} \Subset \Omega$ is normalized. Denote by $\left|D^{2} u\right|$ the largest eigenvalue of the Hessian matrix $D^{2} u$. The $W^{2,1}$ estimate is straightforward by integration by parts

$$
\int_{S_{1}^{0}}\left|D^{2} u(x)\right| d x \leq \int_{S_{1}^{0}} \triangle u(x) d x \leq \int_{\partial S_{1}^{0}} \partial_{\nu} u \leq C_{3},
$$

where $\nu$ is the unit outer normal of $\partial S_{1}^{0}$, the first inequality holds since $u$ is convex and the last inequality follows from the interior estimate of $D u$.

Inequality (5.3) implies the following density estimate (comparing to Assumption $\mathrm{H}$ in $\S 3$ ): there exists a large constant $N>0$ depending only on $n, C_{1}$ and $C_{2}$ such that

$$
\left|D=\left\{x \in S_{1 / K}^{0}: D^{2} u(x)>2 N^{-1} I\right\}\right| \geq \frac{1}{N^{n-1}} \int_{S_{1}^{0}}\left|D^{2} u\right|,
$$

where $|\cdot|$ denotes the Lebesgue measure and $K$ is the constant in the covering lemma.

To prove (5.4), note that at $x \in D$, by equation (1.1) and assumption

$$
\left|D^{2} u(x)\right|<C_{4} N^{n-1} \quad \text { for a constant } C_{4}=C_{3}\left(n, C_{1}, C_{2}\right) .
$$

Let $A=S_{1 / K}^{0}-D=\left\{x \in S_{1 / K}^{0}:\left|D^{2} u(x)\right| \geq C_{4} N^{n-1}\right\}$. By contradiction, if (5.4) is not true, then $|A|>\left|S_{1 / K}^{0}\right|-\frac{1}{N^{n-1}} \int_{S_{1}^{0}}\left|D^{2} u\right|$. Since

$$
C_{4} N^{n-1}|A| \leq \int_{S_{1 / K}^{0}}\left|D^{2} u(x)\right| d x,
$$

from (5.3) we obtain

$$
C_{4} N^{n-1}\left|S_{1 / K}^{0}\right|<\int_{S_{1 / K}^{0}}\left|D^{2} u(x)\right| d x+C_{4} \int_{S_{1}^{0}}\left|D^{2} u(x)\right| d x \leq\left(1+C_{4}\right) C_{3} .
$$

This gives a contradiction by choosing $N$ large.

Choosing a larger constant $N_{0} \geq C_{4} N^{n-1},(5.4)$ implies that

$$
\int_{S_{1}^{0}}\left|D^{2} u(x)\right| d x \leq N_{0}\left|\left\{x \in S_{1 / K}^{0}: N_{0}^{-1} I \leq D^{2} u(x) \leq N_{0} I\right\}\right| .
$$

We remark that by subtracting a linear function, one can obtain (5.5) in any normalized sub-level set $S_{1, u}^{0}(y) \subset \Omega^{\prime} \Subset \Omega$.

2. Recall the definitions of "good" sets $D_{k}$ and "bad" sets $A_{k}$ in $\S 3.2$. In this section we write them in a slightly different form. For a large constant $M>0$ which will be determined later, define

$$
\begin{aligned}
& A_{k}=\left\{x \in S_{1}^{0}:\left|D^{2} u(x)\right| \geq M^{k}\right\}, \\
& D_{k}=S_{1}^{0}-A_{k}=\left\{x \in S_{1}^{0}:\left|D^{2} u(x)\right|<M^{k}\right\},
\end{aligned}
$$

where $k=1,2, \cdots$. Note that when $M=C N^{n-1}$ with $N$ in Assumption $\mathrm{H}$, definition (5.6) is the same as in $\S 3.2$. 
Denote $A_{k}^{\prime}=A_{k}-A_{k+1}$ for $k=1,2, \cdots$. Observe that $M^{k} \leq\left|D^{2} u\right|<$ $M^{k+1}$ in $A_{k}^{\prime}$. From the proof of Theorem 4.1 one can see that the polynomial decay of measures

$$
M^{k}\left|A_{k}^{\prime}\right|<\frac{C}{M^{k(p-1)}}
$$

implies $u \in W^{2, p^{\prime}}$ for all $p^{\prime}<p$. Similarly, for some $\varepsilon>0$

$$
M^{k}\left|A_{k}^{\prime}\right|<\frac{C}{M^{k \varepsilon}}
$$

implies $u \in W^{2,1+\varepsilon^{\prime}}$ for all $\varepsilon^{\prime}<\varepsilon$. In fact,

$$
\begin{aligned}
\int_{S_{1}^{0}}\left|D^{2} u\right|^{1+\varepsilon^{\prime}} & \leq \sum_{k} M^{(k+1)\left(1+\varepsilon^{\prime}\right)}\left|A_{k}^{\prime}\right| \\
& \leq C M^{1+\varepsilon^{\prime}} \sum_{k} M^{k\left(\varepsilon^{\prime}-\varepsilon\right)} \leq C^{\prime} .
\end{aligned}
$$

3. We claim that (5.8) can be deduced from the following induction formula

$$
\int_{A_{k+1}}\left|D^{2} u\right| \leq \tau \int_{A_{k}}\left|D^{2} u\right| \quad \text { for a constant } \tau \in(0,1) .
$$

To see this, by (5.3) $\int_{A_{1}}\left|D^{2} u\right| \leq \tilde{C}$, thus (5.10) implies that

$$
\int_{A_{k}}\left|D^{2} u\right| \leq \tilde{C} \tau^{-1} \tau^{k} \leq C \tau^{k} \quad \text { for } k \geq 1 .
$$

Let $\varepsilon=\log \tau^{-1} / \log M$. Noting that $M^{k}\left|A_{k}^{\prime}\right|<\int_{A_{k}}\left|D^{2} u\right|$, then we obtain the desired estimate (5.8) from (5.10).

4. Therefore, it suffices to prove (5.10). The proof is a modification of that of Lemma 4.1. For each $y \in A_{k+1}$, let

$$
\hat{h}_{y}=\inf \left\{h>0: S_{h}^{0}(y) \subset B \sqrt{N_{0} M^{k} h}(y)\right\},
$$

where $N_{0}$ is the constant in (5.5). By our definition of $A_{k+1}, \hat{h}_{y}>0$ for any $y \in A_{k+1}$. The family $\left\{S_{\hat{h}_{y}, u}^{0}(y)\right\}$ is obviously a covering of $A_{k+1}$. By the covering lemma, there exists a sequence $\left\{y_{i} \in A_{k+1}\right\}$ such that $A_{k+1} \subset \bigcup S_{i}$ and $\left\{S_{i}^{\prime}\right\}$ are mutually disjoint, where $S_{i}=S_{\hat{h}_{y_{i}}, u}^{0}\left(y_{i}\right)$ and $S_{i}^{\prime}=S_{\hat{h}_{y_{i}} / K, u}^{0}\left(y_{i}\right)$.

For each $i$, make a normalization

$$
\tilde{x}=\frac{1}{\hat{h}_{y_{i}}^{1 / 2}} T x, \quad \tilde{u}=\frac{1}{\hat{h}_{y_{i}}} u,
$$

where $T$ is an $n \times n$ matrix such that $S_{1, \tilde{u}}^{0}=\hat{h}_{y_{i}}^{-1 / 2} T\left(S_{i}\right)$ is normalized. By (5.11), $|T| \leq \sqrt{N_{0} M^{k}}$. Since $D^{2} u=T^{t} \circ D^{2} \tilde{u} \circ T$, we have $\left|D^{2} u\right| \leq|T|^{2}\left|D^{2} \tilde{u}\right|$ and

$$
M^{k} \leq\left|D^{2} u(x)\right| \leq N_{0}^{2} M^{k}, \quad \text { if } \quad N_{0}^{-1} I \leq D^{2} \tilde{u}(\tilde{x}) \leq N_{0} I
$$


Applying (5.5) to $\tilde{u}$ in $S_{1, \tilde{u}}^{0}$,

$$
\int_{S_{1, \tilde{u}}^{0}}\left|D^{2} \tilde{u}(\tilde{x})\right| d \tilde{x} \leq N_{0}\left|\tilde{x} \in S_{1 / K, \tilde{u}}^{0}: N_{0}^{-1} I \leq D^{2} \tilde{u}(\tilde{x}) \leq N_{0} I\right| .
$$

By rescaling back,

$$
\begin{aligned}
& \frac{1}{N_{0} M^{k}} \frac{|\operatorname{det} T|}{\hat{h}_{i}^{n / 2}} \int_{S_{i}}\left|D^{2} u(x)\right| d x \\
& \quad \leq N_{0} \frac{|\operatorname{det} T|}{\hat{h}_{i}^{n / 2}}\left|\left\{x \in S_{i}^{\prime}: M^{k} \leq\left|D^{2} u(x)\right| \leq N_{0}^{2} M^{k}\right\}\right|,
\end{aligned}
$$

thus we obtain

$$
\int_{S_{i}}\left|D^{2} u(x)\right| d x \leq N_{0}^{2} M^{k}\left|\left\{x \in S_{i}^{\prime}: M^{k} \leq\left|D^{2} u(x)\right| \leq N_{0}^{2} M^{k}\right\}\right|,
$$

Since $A_{k+1} \subset \bigcup S_{i}$ and $\left\{S_{i}^{\prime}\right\}$ are mutually disjoint, setting $M=N_{0}^{2}$ and summing over $i$ we have

$$
\begin{aligned}
\int_{A_{k+1}}\left|D^{2} u(x)\right| d x & \leq N_{0}^{2} M^{k}\left|\left\{x \in S_{1}^{0}: M^{k} \leq\left|D^{2} u(x)\right| \leq M^{k+1}\right\}\right| \\
& \leq N_{0}^{2} \int_{A_{k} \backslash A_{k+1}}\left|D^{2} u(x)\right| d x .
\end{aligned}
$$

Adding $N_{0}^{2} \int_{A_{k+1}}\left|D^{2} u\right|$ to both sides, we finally obtain

$$
\int_{A_{k+1}}\left|D^{2} u\right| \leq \frac{N_{0}^{2}}{1+N_{0}^{2}} \int_{A_{k}}\left|D^{2} u\right| .
$$

Therefore, (5.10) is proved for the constant $\tau=\frac{N_{0}^{2}}{1+N_{0}^{2}}<1$. Theorem 5.1 is then proved by a standard covering argument.

\section{Monge-Ampère equations of general form}

In this section we consider the Monge-Ampère equation

$$
\operatorname{det}\left[D^{2} u-A(x, u, D u)\right]=f(x, u, D u)
$$

arising in optimal transportation $[\mathbf{2 7}]$ and reflector antenna design $[\mathbf{3 6}, \mathbf{2 0}]$. It is known that the optimal mapping $T=T_{u}(x)$ is determined by a potential function $u$ by

$$
D u(x)=D_{x} c(x, T),
$$

where $c(x, y)$ is the cost function. Differentiating the above formula gives

$$
D^{2} u(x)=D_{x}^{2} c(x, T)+D_{x y}^{2} c \cdot D T .
$$

Hence

$$
\operatorname{det}\left[D^{2} u-D_{x}^{2} c\right]=\left|\operatorname{det} D_{x y}^{2} c\right||\operatorname{det} D T|=\left|\operatorname{det} D_{x y}^{2} c\right| \frac{\rho}{\rho^{*}(T)},
$$


where $\rho, \rho^{*}$ are two probability densities over the initial domain $\Omega$ and the target domain $\Omega^{*}$, respectively. Hence the potential function $u$ satisfies equation (6.1) with the matrix $A$ and the right hand side $f$ given by

$$
\begin{aligned}
& A(x, D u)=D_{x}^{2} c\left(x, T_{u}(x)\right), \\
& f=\left|\operatorname{det} D_{x y}^{2} c\right| \frac{\rho(x)}{\rho^{*}(T)} .
\end{aligned}
$$

We say a solution $u$ of (6.1) is elliptic if the matrix $\left\{D^{2} u-A\right\}$ is positive definite. The potential function is an elliptic solution due to its $c$-convexity, namely at each point $x_{0} \in \Omega$ there exists a $c$-support of $u$ of the form

$$
\varphi(x)=c\left(x, y_{0}\right)+a_{0},
$$

where $a_{0}$ is a constant and $y_{0} \in \mathbb{R}^{n}$ such that

$$
u\left(x_{0}\right)=\varphi\left(x_{0}\right), \quad u(x) \geq \varphi(x), \quad \forall x \in \Omega .
$$

A notion of $c$-convexity of domains was introduced in [27]: a set $U \subset \mathbb{R}^{n}$ is $c$-convex with respect to another set $V \subset \mathbb{R}^{n}$ if the image $D_{y} c(U, y)$ is convex for any $y \in V$. Similarly, $V$ is called $c^{*}$-convex with respect to $U$ if $D_{x} c(x, V)$ is convex for any $x \in U$.

In the special case when $c(x, y)=x \cdot y$, the matrix $A \equiv 0$, equation (6.1) reduces to the standard Monge-Ampère equation (1.1), the notion of $c$-convexity coincides with that of convexity, and a $c$-support is just a support hyperplane.

When $\rho, \rho^{*}>0, \rho \in C^{1,1}(\Omega), \rho^{*} \in C^{1,1}\left(\Omega^{*}\right)$, and $\Omega^{*}$ is $c^{*}$-convex with respect to $\Omega$, the $C^{3}$ regularity of potentials has been established in $[\mathbf{2 7}]$ under the following assumptions:

(A1) For any $x, p \in \mathbb{R}^{n}$, there is a unique $y \in \mathbb{R}^{n}$ such that $D_{x} c(x, y)=p$; and for any $y, q \in \mathbb{R}^{n}$, there is a unique $x \in \mathbb{R}^{n}$ such that $D_{y} c(x, y)=q$.

(A2) For any $(x, y) \in \mathbb{R}^{n} \times \mathbb{R}^{n}, \operatorname{det}\left\{D_{x y}^{2} c(x, y)\right\} \neq 0$.

(A3) For any $x, p \in \mathbb{R}^{n}$, and any $\xi, \eta \in \mathbb{R}^{n}$ with $\xi \perp \eta$,

$$
A_{i j, k l}(x, p) \xi_{i} \xi_{j} \eta_{k} \eta_{l}>0,
$$

where $A_{i j, k l}=D_{p_{k} p_{l}}^{2} A_{i j}$ and $A$ is given by (6.2).

If the $C^{1,1}$ smoothness of $\rho, \rho^{*}$ is removed, the $C^{1}$ regularity and strict $c$-convexity of $u$ has also been obtained in [34]; and the $C^{1, \alpha}$ regularity obtained in $[\mathbf{2 2}, \mathbf{2 5}]$. Hence one can define the sub-level set $S_{h, u}^{0}$ of $u$, namely

$$
S_{h, u}^{0}\left(x_{0}\right)=\{x \in \Omega: u(x)<\varphi(x)+h\},
$$

where $h>0$ is a constant, $\varphi$ is a $c$-support of $u$ at $x_{0}$. Under assumptions (A1)-(A3), it was proved [22] that $S_{h}^{0}=S_{h, u}^{0}\left(x_{0}\right)$ is $c$-convex with respect to $y_{0}$, where $y_{0}=T_{u}\left(x_{0}\right)$, see also [14]. Therefore, by the coordinate transform $x \mapsto D_{y} c\left(x, y_{0}\right)$ we can make $S_{h}^{0}$ convex. By the techniques in Sections 2 and 3 as well as some new estimates, the following results have been obtained. 
TheOREM $6.1([\mathbf{2 3}])$. Let $u \in C^{2}(\Omega)$ be an elliptic solution of $(6.1)$ with $A$ and $f$ given by (6.2) and (6.3), respectively. Assume that (A1)-(A3), $f$ is Dini continuous and satisfies $C_{1} \leq f \leq C_{2}$ for some positive constants $C_{1}, C_{2}$. Then for all $x, y \in \Omega^{\prime} \Subset \Omega$, we have the estimate (3.2). Furthermore, if $f \in C^{\alpha}$ or $C^{0,1}$, we have (3.3) or (3.4), respectively.

TheOREM $6.2([\mathbf{2 4}])$. In the hypotheses of Theorem 6.1, if $f$ is only continuous and $C_{1} \leq f \leq C_{2}$ for some positive constants $C_{1}, C_{2}$, then $D^{2} u \in L^{p}\left(\Omega^{\prime}\right)$ for any $1 \leq p<\infty, \Omega^{\prime} \Subset \Omega$, and we have the estimate (4.3).

The proof of Theorem 6.1 is similar to that of Theorem 3.1. However, one needs to deal with $c$-convex functions instead of convex functions, and to establish an interior $C^{2}$ estimate of Pogorelov type for solutions of (6.1). We refer the readers to $[\mathbf{2 3}]$ for more details.

Theorem 6.2 can be proved using the steps in Section 4, but is more complicated. A crucial part is to understand the local geometry of the potential function and the cost function, in a blow-up process. Namely we show that they converges to quadratic functions in the blowing-up, and that means locally equation (6.1) is very close to the standard Monge-Ampère equation. In other words, we show that equation (6.1) behaves very like the standard Monge-Ampère equation locally. We stress that these properties are established under assumption (A3), or a weak form of it. In general they may not be true. See $[\mathbf{2 4}]$ for a detailed proof.

\section{References}

[1] Brenier, Y., Polar factorization and monotone rearrangement of vector-valued functions. Comm. Pure Appl. Math. 44 (1991), 375-417.

[2] Caffarelli, L.A., Interior a priori estimates for solutions of fully nonlinear equations. Ann. of Math. (2) 130 (1989), 189-213.

[3] Caffarelli, L.A., A localization property of viscosity solutions to the Monge-Ampère equation and their strict convexity. Ann. of Math. (2) 131 (1990), 129-134.

[4] Caffarelli, L.A., Interior $W^{2, p}$ estimates for solutions of Monge-Ampère equations. Ann. of Math. 131 (1990), 135-150.

[5] Caffarelli, L.A., Some regularity properties of solutions to Monge-Ampère equations. Comm. Pure Appl. Math. 44 (1991), 965-969.

[6] Caffarelli, L.A., The regularity of mappings with a convex potential, J. Amer. Math. Soc. 5 (1992), 99-104.

[7] Caffarelli, L.A., Nirenberg, L. \& Spruck, J., Dirichlet problem for nonlinear second order elliptic equations I, Monge-Ampère equations, Comm. Pure Appl. Math. 37 (1984), 369-402.

[8] Calabi, E., Improper affine hyperspheres of convex type and a generalization of a theorem by K. Jörgens, Mich. Math. J. 5 (1958), 105-126.

[9] Cheng, S.Y. \& Yau, S.T., On the regularity of the Monge-Ampère equation $\operatorname{det} \frac{\partial^{2} u}{\partial x_{i} \partial x_{j}}=F(x, u)$, Comm. Pure Appl. Math. 30 (1977), 41-68.

[10] Chou, K.S. \& Wang, X.-J., Entire solutions to Monge-Ampère equations, Comm. Pure Appl. Math. 49 (1996) 529-539.

[11] De Philippis, G. and Figalli, A., $W^{2,1}$ regularity for solutions of the Monge-Ampère equaiton. Invent. Math. 192 (2013), 55-69. 
[12] De Philippis, G., Figalli, A. and Savin, O., A note on interior $W^{2,1+\varepsilon}$ estimates for the Monge-Ampère equation. Math. Ann., 357 (2013), 11-22.

[13] Evans, L.C., Classical solutions of fully nonlinear, convex, second order elliptic equations, Comm. Pure Appl. Math. 25 (1982), 333-362.

[14] Figalli, A., Kim, Y.-H. and McCann, R.J., Hölder continuity and injectivity of optimal maps, Arch. Rat. Mech. Anal., 209 (2013), 747-795.

[15] Gilbarg, D. and Trudinger, N.S., Elliptic partial differential equations of second order. Springer-Verlag, Berlin, 1983.

[16] Gutiérrez, C.E., The Monge-Ampère equation. Progress in Nonlinear Differential Equations and their Applications, 44. Birkhäuser Boston, Inc., Boston, MA, 2001.

[17] Heinz, E., Uber die Differentialungleichung $0<\alpha \leq r t-s^{2} \leq \beta<\infty$. Math. Z. 72 (1959/1960), 107-126.

[18] Huang, Q., Sharp regularity results on second derivatives of solutions to the MongeAmpère equation with VMO type data. Comm. Pure Appl. Math. 62 (2009), 677-705.

[19] Jian, H.Y. and Wang, X.-J., Continuity estimates for the Monge-Ampère equation. SIAM J. Math. Anal. 39 (2007), 608-626.

[20] Karakhanyan, A. and Wang, X.-J., On the reflector shape design, J. Diff. Geom., 84 (2010), 561-610.

[21] Krylov, N.V., Boundedly inhomogeneous elliptic and parabolic equations (Russian), Izv. Akad. Nauk SSSR Ser. Mat. 46 (1982), 487-523. English translation in Math. USSR. Izv. 20 (1983), 459-492.

[22] Liu, J., Hölder regularity of optimal mappings in optimal transportation. Calc. Var. PDE. 34 (2009), 435-451.

[23] Liu, J., Trudinger, N.S. and Wang, X.-J., Interior $C^{2, \alpha}$ regularity for potential functions in optimal transportation. Comm. Partial Differential Equations, 35 (2010), 165-184.

[24] Liu, J., Trudinger, N.S. and Wang, X.-J., On asymptotic behaviour and $W^{2, p}$ regularity of potentials in optimal transportation, Arch. Rat. Mech. Anal., 215 (2015), 867-905.

[25] Loeper, G., On the regularity of solutions of optimal transportation problems. Acta Math. 202 (2009), 241-283.

[26] Loeper, G., Regularity of optimal maps on the sphere: the quadratic cost and the reflector antenna, Arch. Ration. Mech. Anal. 199 (2011), 269-289.

[27] Ma, X.N., Trudinger, N.S. and Wang, X.-J., Regularity of potential functions of the optimal transportation problem. Arch. Rat. Mech. Anal., 177 (2005), 151-183.

[28] Nikolaev, I.G. \& Shefel, S.Z., Convex surfaces with positive bounded specific curvature, and a priori estimates for Monge-Ampère equations (Russian), Sibirsk. Mat. Zh. 26 (1985), 120-136.

[29] Pogorelov, A.V., The Minkowski Multidimensional Problem. Scripta Series in Mathematics. New York: J.Wiley, 1978.

[30] Schmidt, T., $W^{2,1+\varepsilon}$ estimate for the Monge-Ampère equation. Adv. Math., 240 (2013), 672-689.

[31] Schneider, R., Convex bodies: The Brunn-Minkowski theory, Cambridge University Press, 1993.

[32] Schulz, F., Regularity theory for quasilinear elliptic systems and Monge-Ampère equations in two dimensions, Lecture notes in Math. Volume 1445, 1990.

[33] Trudinger, N.S. and Wang, X.-J., The Monge-Ampère equation and its geometric applications. Handbook of geometric analysis. No. 1, 467-524, Adv. Lect. Math. (ALM), 7, Int. Press, Somerville, MA, 2008.

[34] Trudinger, N.S. and Wang, X.-J., On strict convexity and continuous differentiability of potential functions in optimal transportation. Arch. Rat. Mech. Anal., 192 (2009), 403-418. 
[35] Wang, X.-J., Some counterexamples to the regularity of Monge-Ampère equations, Proc. Amer. Math. Soc. 123 (1995), no. 3, 841-845.

[36] Wang, X.-J., On the design of a reflector antenna, Inverse problems, 12 (1996), 351375

[37] Wang, X.-J., Schauder estimate for elliptic and parabolic equations, Chinese Ann. of Math., Series B, 27 (2006), 637-642.

[38] Wang, X.-J., Lecture Notes at Tsinghua University, Beijing, 2011.

Institute for Mathematics and its Applications, School of Mathematics and Applied Statistics, University of Wollongong, Wollongong, NSW 2522, Australia.

E-mail address: jiakunl@uow.edu.au

Centre for Mathematics and Its Applications, The Australian National University, Canberra, ACT 0200, Australia.

E-mail address: Xu-Jia. Wang@anu.edu.au 